\title{
Wee1 inhibition can suppress tumor proliferation and sensitize p53 mutant colonic cancer cells to the anticancer effect of irinotecan
}

\author{
YUPING YIN ${ }^{1}$, QIAN SHEN ${ }^{2}$, RUIKANG TAO ${ }^{3}$, WEILONG CHANG $^{4}$, RUIDONG LI $^{1}$, \\ GENGCHEN XIE $^{1}$, WEIZHEN LIU ${ }^{1}$, PENG ZHANG ${ }^{1}$ and KAIXIONG TAO ${ }^{1}$
}

\begin{abstract}
Departments of ${ }^{1}$ Gastrointestinal Surgery and ${ }^{2}$ Oncology, Union Hospital, Tongji Medical College, Huazhong University of Science and Technology, Wuhan, Hubei 430022, P.R. China; ${ }^{3}$ Center for Biomolecular Science and Engineering, University of California Santa Cruz, Santa Cruz, CA 95064, USA; ${ }^{4}$ Department of Gastrointestinal Surgery, The First Affiliated Hospital of Zhengzhou, Zhengzhou, Henan 450000, P.R. China
\end{abstract}

Received November 30, 2016; Accepted August 16, 2017

DOI: $10.3892 / \mathrm{mmr} .2017 .8230$

\begin{abstract}
Wee1 is an oncogenic nuclear kinase, which can regulate the cell cycle as a crucial G2M checkpoint. Overexpression of Weel can be observed in various cancer types, which may lead to a poor prognosis, but the potential therapeutic value of Weel in colorectal cancer has not been fully studied. In the present study, the role of Weel in colonic cancer was investigated. Weel inhibition by small interfering RNA was demonstrated to significantly restrain cancer cell proliferation and sensitize the p53 mutant colonic cancer cell lines HT29 and SW480 to the effect of treatment with ionizing radiation. The anticancer effect of the Weel inhibitor MK1775 was investigated in these two colonic cancer cell lines. MK1775 was demonstrated to induce significant DNA damage, suppress cell viability and induce apoptosis. In addition, MK1775 sensitized HT29 and SW480 cells to the effect of irinotecan. Annexin V/propidium iodide staining demonstrated that combination therapy can induce increased apoptosis compared with MK1775 or irinotecan monotherapy. The results of western blot analysis also indicated increased expression of the DNA damage marker histone $\mathrm{H} 2 \mathrm{AX}$, and apoptosis-associated protein cleaved caspase 3, in HT29 and SW480 cells. In conclusion, the present study indicated that Weel may be a valuable target for treatment of p53 mutant colonic cancer.
\end{abstract}

\section{Introduction}

Colorectal cancer is one of the most prevalent malignant diseases, and is the fourth highest cancer-associated mortality

Correspondence to: Dr Kaixiong Tao or Dr Peng Zhang, Department of Gastrointestinal Surgery, Union Hospital, Tongji Medical College, Huazhong University of Science and Technology, 1277 Jiefang Avenue, Wuhan, Hubei 430022, P.R. China

E-mail: tao_kaixiong@163.com

E-mail: zhangpengzs208@163.com

Key words: Wee1, MK1775, irinotecan, colorectal cancer
worldwide(1).Early diagnosis has improved the clinicaloutcome of patients with colorectal cancer, and conventional therapeutic strategies serve a crucial role for advanced colorectal cancer, including surgery, and chemoradiotherapy (2). Due to the poor therapeutic efficiency of traditional cytotoxic drugs, the prognosis of patients with advanced cancer remains poor (3). Therefore, novel targeted drugs and more efficient therapeutic strategies are urgently needed to improve the clinical outcome for patients with advanced colorectal cancer (4).

Important mechanisms in response to DNA damage are the cell cycle checkpoints that can prevent cells entering into mitosis by arresting the cell in the G1 or G2 phase, which allows enough time for DNA repair to maintain genomic integrity (5). The lack of a functional G1 arrest is common in cancer cells due to dysfunction of p53, so cancer cells are largely dependent on the $\mathrm{G} 2$ checkpoint to repair endogenous and exogenous DNA damage; therefore, targeting the G2 checkpoint is a promising strategy for cancer therapy $(6,7)$, especially in p53 mutant cancer cells. Weel is a nuclear kinase, which can regulate the $\mathrm{G} 2$ checkpoint by activating phosphorylation of cyclin dependent kinase 1 (CDK1) at the Tyr15 residue and therefore delaying the entry into mitosis $(8,9)$. Wee1 overexpression occurs in a number of cancer types, including ovarian cancer (10), melanoma (11) and lung cancer (12), and indicates a poor outcome. In light of this promising hypothesis, inhibitors targeting Wee1 have been designed, and a number of clinical trials are underway. Of these inhibitors, MK1775 is one of the most promising candidates, which can potently and selectively inhibit Weel kinase in an adenosine triphosphate-competitive manner, and a number of studies have demonstrated that MK1775 can sensitize cells to the effect of various chemotherapy drugs in solid tumor, including cisplatin, 5-fluorouracil (13-15).

In the field of colorectal cancer, the value of targeting Weel is disputed. On one hand, certain studies have reported that Weel has limited therapeutic value due to its poor correlation with clinical biological characteristics and prognosis (16); however, the Weel inhibitor MK1775 could sensitize p53-deficient colonic cancer cell lines to the anticancer effect of a number of DNA damaging drugs (17). However, other studies have argued that this chemosensitization effect was not dependent on p53 
status (18). Due to this controversy, further evaluation of the potential therapeutic value of Wee1 in colonic cancer is needed. In the present study, the effect of Weel on cell proliferation and the response to ionizing radiation (IR) treatment in HT29 and SW480 cells was investigated. The anticancer effect of the Weel inhibitor MK11775 was analyzed, and whether MK1775 can enhance the efficiency of DNA damage associated drug irinotecan, which is one of the most important chemotherapy reagents used in the clinical treatment of patients with colorectal cancer with metastasis, was further examined.

\section{Materials and methods}

Cell culture. Two human colon cancer cells lines, HT29 and SW480 were purchased from the American Type Culture Collection (Manassas, VA, USA) and cultured with RMPI-1640 (Gibco; Thermo Fisher Scientific, Inc., Waltham, MA, USA) with $10 \%$ fetal bovine serum (Gibco; Thermo Fisher Scientific, Inc.) supplemented with $1 \%$ penicillin and streptomycin (Gibco; Thermo Fisher Scientific, Inc.). Cells were conventionally maintained in an incubator at $37^{\circ} \mathrm{C}$ with $5 \%$ carbon dioxide and the culture medium was routinely changed every three days.

Small interfering (si)RNA construction and transfection. Weel and non-target control siRNA were purchased from Sigma-Aldrich (Merck KGaA, Darmstadt, Germany), the Wee1 siRNA sequence: 5'-AAUGAUUCCUGUGGUGAA GAC-3'. Non-target control siRNA sequence: 5'-UAAGGC UAUGAAGAGAUAC-3', the final concentration of the siRNA for the experiments was $20 \mathrm{nM}$. Weel and non-target siRNA were transient transfected into cells using Oligofectamine (Invitrogen; Thermo Fisher Scientific, Inc.) for 24 or $48 \mathrm{~h}$ according to the protocol from the manufacturer.

MTT assays. Cells were seeded into 96-wells cell culture plates (3,000 cells/well) overnight, then analyzed under different experimental conditions. A total of $20 \mu 1$ MTT (Invitrogen; Thermo Fisher Scientific, Inc.; $2 \mathrm{mg} / \mathrm{ml}$ ) was added to each well, and cells were and incubated for $4 \mathrm{~h}$. Dimethyl sulfoxide (DMSO) was added to dissolve the crystals, cells were agitated gently for another $5 \mathrm{~min}$, and then the absorbance of each well was detected at $570 \mathrm{nM}$. The MTT assay was used to analyze the effect of Weel on cell proliferation, the response to treatment with IR and to examine the anticancer effect of MK1775, irinotecan and combination therapy in HT29 and SW480 cells.

Radiosensitivity assays. Cell radiosensitivity was detected using an MTT assay. HT29 and SW480 cells were transfected with Weel siRNA for $48 \mathrm{~h}$ and the control groups were transfected with siRNA. Cells were then seeded into a 96-well plate (500 cells/well). Cells were cultured for another $24 \mathrm{~h}$, then IR was administered (dosage, 4 Gy) using an X-ray irradiator (RAD Source, LLC., Brentwood, TN, USA). Cells were cultured for 4 days, then the cell viability in the control and Wee1 knockdown groups with or without IR treatment were detected by MTT assay.

Apoptosis assays. HT29 and SW480 cells were seeded into 6 -well plates $\left(3 \times 10^{5}\right.$ cells/well), to analyze the anticancer effect of MK1775. Cells were treated with $1 \mu \mathrm{M}$ MK1775 for $24 \mathrm{~h}$, to investigate whether MK1775 can sensitize cells to the effect of irinotecan. Cells were divided into four groups: Control (DMSO), MK1775 monotherapy, irinotecan monotherapy, and combination. Following treatment for $24 \mathrm{~h}$, cell were harvested, washed with cold PBS twice, and Annexin-V (Invitrogen; Thermo Fisher Scientific, Inc.) and propidium iodide (Sigma-Aldrich; Merck KGaA) were added. Cells were then incubated for $10 \mathrm{~min}$ at room temperature in the dark, and the apoptosis rate in each group was detected using flow cytometry (Beckman Coulter, Inc., Brea, CA, USA and analysed by Kaluza ${ }^{\circledR}$ software version 1.2 (Beckman Coulter, Inc.).

Western blot analysis. Cells were harvested and lysed in a radioimmunoprecipitation assay lysis buffer with protease and phosphorylation inhibitor cocktails (GenDepot, Inc., Barker, TX, USA). The protein concentration for each group was determined by Bradford protein assay. A total of $30 \mu \mathrm{g}$ protein for each sample was loaded onto a gradient SDS-PAGE gel. Following gel electrophoresis, the proteins were transferred to polyvinyl difluoride membranes, then blocked with 5\% skimmed milk (Sigma-Aldrich; Merck KGaA) at room temperature for $1 \mathrm{~h}$. Subsequently, the membranes were washed with PBS with 0.1\% Tween-20 (PBST) three times, then incubated overnight at $4^{\circ} \mathrm{C}$ with primary antibodies: Anti-Weel (1:1,000; cat no. 4936), anti-phospho-CDK1 (Thr 14/Tyr15 (1:1,000; cat no. 4539), anti-cleaved-caspase 3 (1:1,000; cat no. 9661) and anti-r-histone $\mathrm{H} 2 \mathrm{AX}$ (H2AX; 1:1,000; cat no. 7631) were purchased from Cell Signaling Technology, Inc., (Danvers, MA, USA); anti-total CDK1 (1:500; cat no. sc-53219) was purchased from Santa Cruz Biotechnology, Inc., (Dallas, TX, USA) and anti- $\beta$-actin (1:100,000; cat no. A1978) was purchased from Sigma-Aldrich (Merck KGaA). $\beta$-actin was used as a loading control. Anti mouse or rabbit-horse radish peroxidase conjugated secondary antibodies (cat nos. 32260 and 32230; Invitrogen; Thermo Fisher Scientific, Inc.; 1:2,500 in PBST) were incubated with the membranes at room temperature for $1 \mathrm{~h}$, following washing with PBST three times. The expression of target proteins was detected using enhanced chemiluminescence solution (Bio-Rad Laboratories, Inc., Hercules, CA, USA).

Statistical analysis. All data were presented as the mean \pm standard deviation and each experiment was performed three times. The paired Student's t-test was used to calculate the statistical value for the difference between two groups, and multigroup comparisons were performed using one way analysis of variance followed by the Student-Newman-Keuls post hoc test using GraphPad Prism version 6.0 software (GraphPad Software, Inc., La Jolla, CA, USA). P<0.05 was considered to indicate a statistically significant difference.

\section{Results}

Weel ablation could impair the proliferative ability of the cell and sensitize p53 mutant colonic cancer cell lines, HT29 and SW480 to the effect of IR treatment. To examine the role of Weel in colonic cancer cells, HT29 and SW480 cells were transfected with Weel siRNA for 48 h (Fig. 1). Weel knockdown efficiency was examined by western blotting, 
A
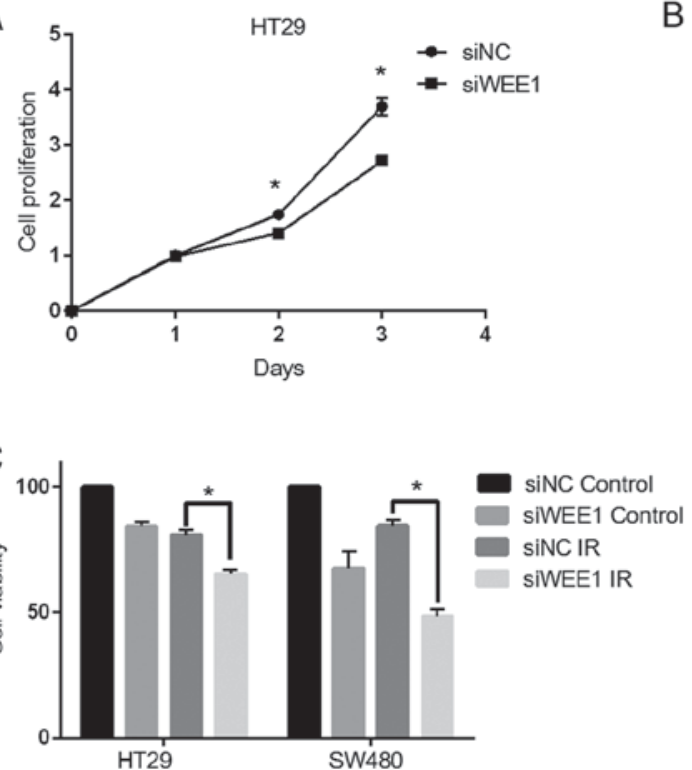

B

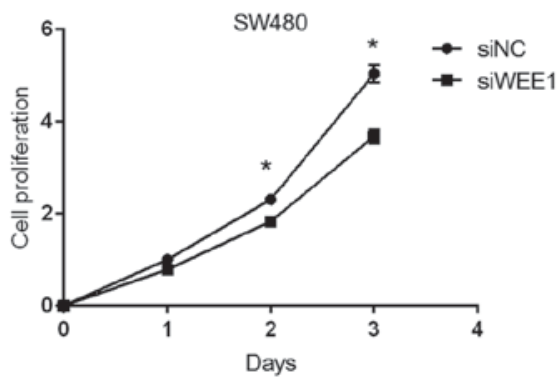

D

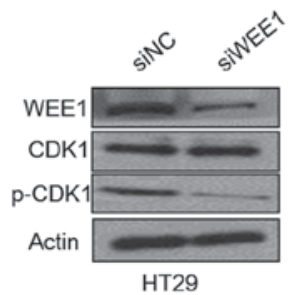

Figure 1. Wee1 knockdown by siRNA reduces cell proliferation and sensitizes colonic cancer cells to treatment with IR. HT29 and SW480 cells were transfected with siNC and Wee1 siRNA. Cell proliferation on days 1, 2 and 3 following transfection were measured by MTT assay in (A) HT29 and (B) SW480 cells. (C) HT29 and SW480 cells were exposed to IR treatment with a dosage of 4 Gy, then cultured for another 4 days, and cell viability were detected by MTT assays. (D) In HT29 and SW480 cells, the expression of Wee1, total CDK1, p-CDK1 (Ty15) in the control and Wee1 knockdown groups were detected by western blotting. Data are presented as the mean \pm SD. ${ }^{*} \mathrm{P}<0.05$ vs. siNC. IR, ionizing radiation; si, small interfering; CDK1, cyclin dependent kinase; NC, non-target control; p, phosphorylated.

as exhibited in Fig. 1D. Weel expression was decreased in HT29 and SW480 cells, and it was also demonstrated that phosphorylation at the Tyr15 residue of CDK1 was inhibited. An MTT assay was used to examine cell proliferation and the sensitivity of cells to treatment with IR; Weel knockdown was demonstrated to significantly suppress the proliferation of HT29 ( $\mathrm{P}<0.05$; Fig. 1A) and SW480 (P<005; Fig. 1B) cells. The effect of Wee1 silencing by siRNA on cell viability during treatment with IR was investigated, and the sensitivity of cells to IR treatment was increased compared with the untreated cells (Fig. 1C).

Weel inhibitor MK1775 can significantly induce DNA damage, leading to apoptosis and decreased cell viability in HT29 and SW480 cells. Nearly 40 clinical trials of the Wee1 inhibitor MK1775 are now underway (19), but its anticancer effect and the potential molecular alterations are not well studied in colorectal cancer cells. From the results of the MTT assay exhibited in Fig. 2A, MK1775 can decrease cell viability in HT29 and SW480 cells. The $\mathrm{IC}_{50}$ following treatment for 4 days was $0.376 \pm 0.134 \mu \mathrm{M}$ in HT29 and $0.739 \pm 0.214 \mu \mathrm{M}$ in SW480 cells. The apoptosis assays also demonstrated that MK1775 could induce cell apoptosis in these two colonic cancer cell lines (Fig. 2B). Western blotting was used to detect the molecular alterations induced by MK1775 in HT29 and SW480 cells (Fig. 2C and D, respectively). MK1775 decreased the level of Wee1 and the phosphorylation of CDK1, and it also was observed that the DNA damage marker $\gamma$-H2AX and apoptosis marker cleaved-caspase 3 increased in a dose-dependent manner (Fig. 2C and D).

Weel inhibitor MK1775 could sensitize p53 mutant colonic cancer cells to the effect of irinotecan. Although MK1775 demonstrated a potent anticancer effect in colonic cancer cells, it remains a better choice to use it in combination with other DNA damaging drugs for cancer treatment, because combination strategy can decrease the effective dosage for each drug, and it also can delay the development of drug resistance. Irinotecan is one of most important chemotherapy drugs, which is widely used for advanced colorectal cancer treatment (20), especially for fluorouracil resistant patients. In the present study, whether MK1775 could enhance the therapeutic efficiency of irinotecan in HT29 and SW480 cells was investigated. The results revealed that irinotecan combined with MK1775 decreased cell viability compared with irinotecan treatment alone (Fig. 3A and B). Additionally, combined treatment induced increased levels of apoptosis, compared with treatment with one drug alone (Fig. 3C and D).

Weel inhibitor MK1775 combined with irinotecan could induce an increased level of DNA damage associated protein $\gamma$-H2AX and apoptosis marker cleaved-caspase 3 in HT29 and SW480 cells. Along with investigating the potency of MK1775 and irinotecan combination in HT29 and SW480 cells, the molecular mechanisms were also analyzed using western blotting. As presented in Fig. 4A and B, irinotecan can induce phosphorylation of CDK1, which may serve a role in aiding cancer cells in escaping from the anticancer effect of irinotecan, and MK1775 was also demonstrated to decreased the phosphorylation of CDK1. And in the combination treatment groups, higher levels of DNA damage associated protein $\gamma$-H2AX and apoptosis marker cleaved-caspase 3 were observed in both HT29 and SW480 cells. The results of the present study support the hypothesis that MK1775 could enhance the anticancer effect of irinotecan on p53 mutant colonic cancer cells. 


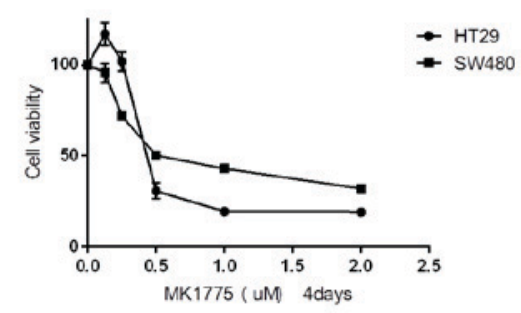

C

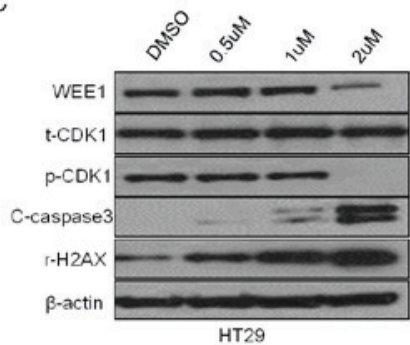

B
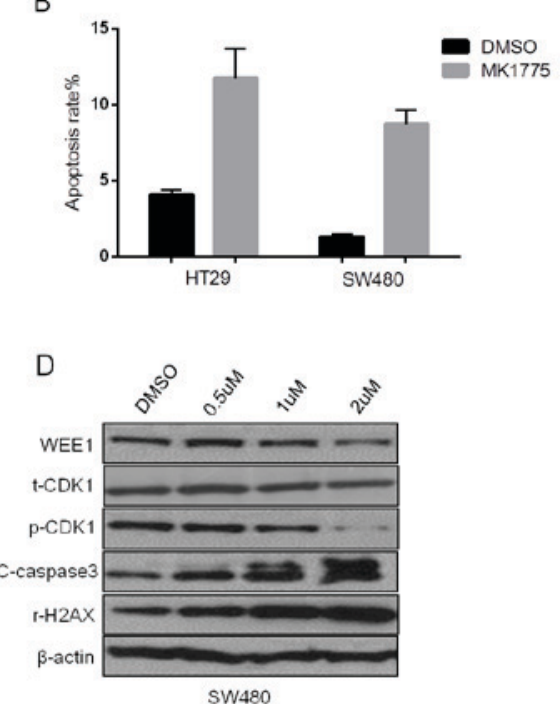

Figure 2. Wee1 inhibitor MK1775 monotherapy demonstrates an anticancer effect in colonic cancer cell lines. HT29 and SW480 cells were treated with Wee1 inhibitor MK1775. (A) HT29 and SW480 cells were treated with $0.5,1$ and $2 \mu$ M MK1775 for 4 days, MTT assay was used to detect the cell viability. (B) Cells were treated with $1 \mu \mathrm{M}$ MK1775 for $24 \mathrm{~h}$, Annexin-V/propidium iodide staining were performed to detected cell apoptosis in HT29 and SW480 cells. Representative western blot images of (C) HT29 and (D) SW480 cells incubated with 0.5, 1 and $2 \mu \mathrm{M}$ MK1775 for $24 \mathrm{~h}$. Data are presented as the mean \pm SD. CDK1, cyclin dependent kinase; H2AX, histone H2AX; p, phosphorylated; t-, total; DMSO, dimethyl sulfoxide.

A

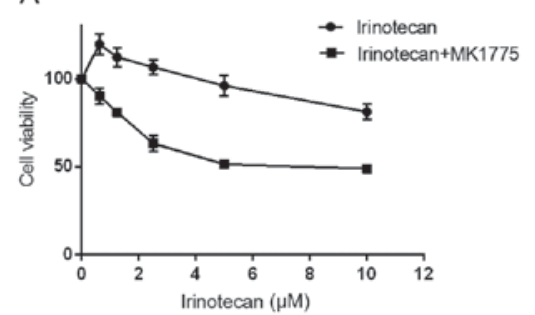

C

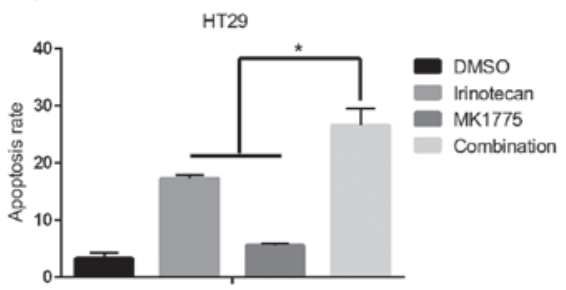

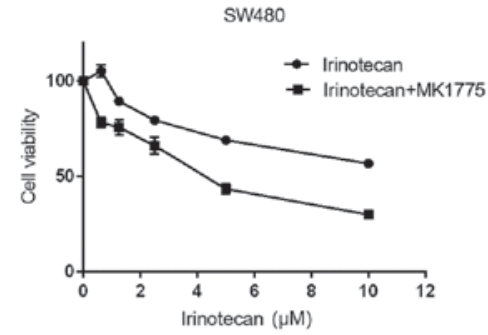

D

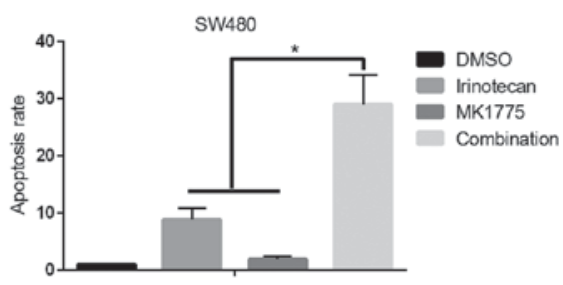

Figure 3. MK1775 can sensitize colonic cancer cells to the anticancer effect of irinotecan. Cell viability in (A) HT29 and (B) SW480 cells, as assessed by MTT assay. Apoptosis rates in (C) HT29 and (D) SW480 cells treated with MK1775, irinotecan, a combination of the two or a DMSO control. Data are presented as the mean \pm SD. ${ }^{*} \mathrm{P}<0.05$. DMSO, dimethyl sulfoxide
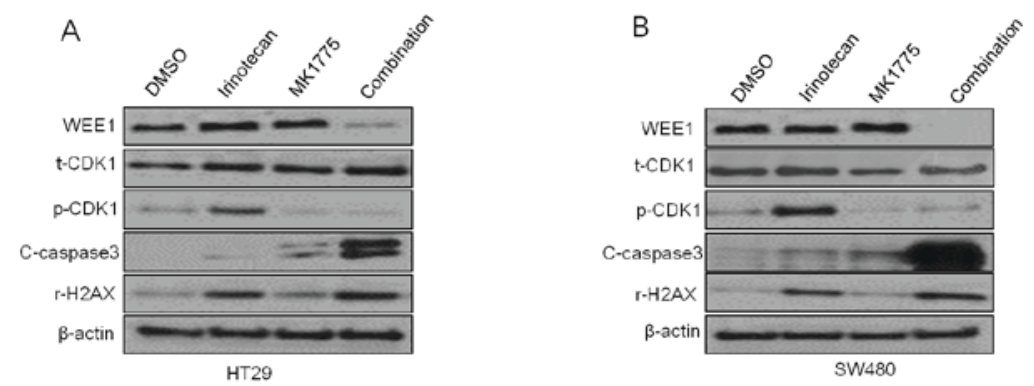

Figure 4. Wee1 inhibitor MK1775 combined with irinotecan can induce an increase in DNA damage associated protein $\gamma$-H2AX and apoptosis marker c-caspase 3 in HT29 and SW480 cells. Representative western blot images of protein expression levels of Wee1, CDK1, p-CDK1, c-caspase 3, H2AX and the actin control to treatment with irinotecan, MK1775, a combination of the two or DMSO control in (A) HT29 and (B) SW480 cells. CDK1, cyclin dependent kinase 1; p-, phosphorylated; H2AX, histone H2AX; c-, cleaved; t-total; DMSO, dimethyl sulfoxide. 


\section{Discussion}

In normal cells, DNA damage usually is repaired by arresting the cell at the G1 phase, but in tumor cells (20), due to the frequency of G1 checkpoint deficiencies, especially in p53 deregulated cells, the $\mathrm{G} 2$ checkpoint serves a crucial role in DNA damage repair $(21,22)$. As a result of this, targeting the effectors, which are involved in G2 checkpoints, is considered a potent strategy for cancer therapy. Unlike other strategies, which aim to block cell cycle processes, Weel inhibition can speed up cell cycle progression, promoting increased entry into mitosis with unrepaired DNA damage, ultimately leading to apoptosis and cell death. Therefore, it is reasonable that Wee1 inhibition can potentiate the anticancer effect of a number of conventional DNA damaging drugs. However, there remains some controversy about the chemosensitization effect of Weel inhibition, with certain studies reporting that Weel inhibition can only enhance the effect of DNA damage-associated agents and radiotherapy in 553 deficient cancer cells $(17,18)$, but others argued that Weel inhibition demonstrated a chemosensitization effect in both p53 wild and mutant cancer cells $(18,23)$.

The results of the present study demonstrated that Wee1 knockdown can inhibit tumor cell proliferation in p53 mutant colonic cancer cell lines HT29 and SW480. This may be due to the attenuated capacity of the cell to repair endogenous DNA damage from the loss of the G2 checkpoint by Weel inhibition, and Weel ablation may also increase the effect of treatment with IR. To further investigate the potential clinical value of the results of the present study, the anticancer effect of the Weel inhibitor MK1775 in HT29 and SW480 cells was analyzed. Logically, Weel inhibition ought to have an improved anticancer effect in p53 mutant cells due to the lack of G1 arrest, and the data from the present study demonstrated that MK1775 could significantly inhibit cell proliferation and induce apoptosis in these two p53 mutant colon cancer cell lines. The results of the present study were in accordance with data published in previous studies in other cancer types $(13,24)$, and western blotting also demonstrated that MK1775 could increase the DNA damage marker $\gamma$-H2AX and apoptosis associated protein cleaved caspase 3 in dose-dependent manner. Although it seems that MK1775 monotherapy could achieve an anticancer effect in p53 mutant colonic cancer cells, the dosage for treatment is still a challenge, as the toxicity of agents targeting the cell cycle checkpoint is big problem. Combination with other chemotherapy drugs is a better choice as a first-line chemotherapy regime in metastatic colonic cancer (25), and a number of studies are seeking the proper strategy to increase the sensitivity of cancer cells to the cytotoxicity effect (26-28). In the present study MK1775 was demonstrated to increase the sensitivity of cells to the effect of irinotecan both in HT29 and SW480, and resulting in increased $\gamma$-H2AX and cleaved-caspase 3 .

In conclusion, although negative results have been reported regarding the association between Weel expression and clinical outcome, Weel is still a valuable target in patients with p53 mutant colorectal cancer. Weel inhibition can decrease cell viability in colorectal cancer cell lines, furthermore, it was demonstrated that the Weel inhibitor MK1775 is a potent anticancer drugs and can induce DNA damage in colorectal cancer cells. In addition, MK1775 has a chemosensitization effect on treatment with irinotecan in colorectal cancer cells, which may help to increase the efficiency of treatment with irinotecan, enabling improved clinical outcomes in patients with advanced colorectal cancer.

\section{Acknowledgements}

The present study was supported by the National Natural Science Foundation of China (grant no. 81572413), the Natural Science Foundation of Hubei Province (grant no. 2015CFB378), the Research Fund of Public welfare in Health, Industry Health and Family Planning Committee of China (grant no. 201402015).

\section{References}

1. Miller KD, Siegel RL, Lin CC, Mariotto AB, Kramer JL, Rowland JH, Stein KD, Alteri R and Jemal A: Cancer treatment and survivorship statistics, 2016. CA Cancer J Clin 66: 271-289, 2016.

2. Zhou Y, Abel GA, Hamilton W, Pritchard-Jones K, Gross CP, Walter FM, Renzi C, Johnson S, McPhail S, Elliss-Brookes L and Lyratzopoulos G: Diagnosis of cancer as an emergency: A critical review of current evidence. Nat Rev Clin Oncol 14: 45-56, 2017.

3. Kolligs FT: Diagnostics and epidemiology of colorectal cancer. Visc Med 32: 158-164, 2016.

4. Moriarity A, O'Sullivan J, Kennedy J, Mehigan B and McCormick P: Current targeted therapies in the treatment of advanced colorectal cancer: A review. Ther Adv Med Oncol 8: 276-293, 2016.

5. Kastan MB and Bartek J: Cell-cycle checkpoints and cancer. Nature 432: 316-323, 2004.

6. Santo L, Siu KT and Raje N: Targeting cyclin-dependent kinases and cell cycle progression in human cancers. Semin Oncol 42: 788-800, 2015.

7. Benada J and Macurek L: Targeting the checkpoint to kill cancer cells. Biomolecules 5: 1912-1937, 2015.

8. Russell P and P Nurse: Negative regulation of mitosis by Wee1+, a gene encoding a protein kinase homolog. Cell 49: 559-567, 1987.

9. Gould KL and Nurse P: Tyrosine phosphorylation of the fission yeast $\mathrm{cdc} 2+$ protein kinase regulates entry into mitosis. Nature 342: 39-45, 1989.

10. Leary A, Auguste A and Mesnage S: DNA damage response as a therapeutic target in gynecological cancers. Curr Opin Oncol 28: 404-411, 2016

11. Magnussen GI, Holm R, Emilsen E, Rosnes AK, Slipicevic A and Flørenes VA: High expression of Weel is associated with poor disease-free survival in malignant melanoma: Potential for targeted therapy. PLoS One 7: e38254, 2012.

12. Yoshida T, Tanaka S, Mogi A, Shitara Y and Kuwano H: The clinical significance of Cyclin B1 and Wee1 expression in non-small-cell lung cancer. Ann Oncol 15: 252-256, 2004.

13. Kim HY, Cho Y, Kang H, Yim YS, Kim SJ, Song J and Chun KH: Targeting the WEE1 kinase as a molecular targeted therapy for gastric cancer. Oncotarget 7: 49902-49916, 2016.

14. Hirai H, Iwasawa Y, Okada M, Arai T, Nishibata T, Kobayashi M, Kimura T, Kaneko N, Ohtani J, Yamanaka K, et al: Small-molecule inhibition of Wee1 kinase by MK-1775 selectively sensitizes p53-deficient tumor cells to DNA-damaging agents. Mol Cancer Ther 8: 2992-3000, 2009.

15. Osman AA, Monroe MM, Ortega Alves MV, Patel AA, Katsonis P, Fitzgerald AL, Neskey DM, Frederick MJ, Woo SH, Caulin C, et al: Wee-1 kinase inhibition overcomes cisplatin resistance associated with high-risk TP53 mutations in head and neck cancer through mitotic arrest followed by senescence. Mol Cancer Ther 14: 608-619, 2015.

16. Egeland EV, Flatmark K, Nesland JM, Flørenes VA, Mælandsmo GM and Boye K: Expression and clinical significance of Wee1 in colorectal cancer. Tumour Biol 37: 12133-12140, 2016.

17. Hirai H, Arai T, Okada M, Nishibata T, Kobayashi M, Sakai N, Imagaki K, Ohtani J, Sakai T, Yoshizumi T, et al: MK-1775, a small molecule Weel inhibitor, enhances anti-tumor efficacy of various DNA-damaging agents, including 5-fluorouracil. Cancer Biol Ther 9: 514-522, 2010 . 
18. Cuneo KC, Morgan MA, Davis MA, Parcels LA, Parcels J, Karnak D, Ryan C, Liu N, Maybaum J and Lawrence TS: Weel kinase inhibitor AZD1775 radiosensitizes hepatocellular carcinoma regardless of TP53 mutational status through induction of replication stress. Int J Radiat Oncol Biol Phys 95: 782-790, 2016.

19. Leijen S, van Geel RM, Sonke GS, de Jong D, Rosenberg EH, Marchetti S, Pluim D, van Werkhoven E, Rose S, Lee MA, et al: Phase II study of WEE1 inhibitor AZD1775 plus carboplatin in patients with TP-53 mutated ovarian cancer refractory or resistant to first-line therapy with 3 months. J Clin Oncol 34: 4354-4361, 2016.

20. Wulaningsih W, Wardhana A, Watkins J, Yoshuantari N, Repana D and Van Hemelrijck M: Irinotecan chemotherapy combined with fluoropyrimidines versus irinotecan alone for overall survival and progression-free survival in patients with advanced and/or metastatic colorectal cancer. Cochrane Database Syst Rev 2: CD008593, 2016.

21. Beetham KL and Tolmach LJ: The action of caffeine on X-irradiated hela cells. V. Identity of the sector of cells that expresses potentially lethal damage in G1 and G2. Radiat Res 91: 199-211, 1982.

22. Dixon $\mathrm{H}$ and Norbury CJ: Therapeutic exploitation of checkpoint defects in cancer cells lacking p53 function. Cell Cycle 1: 362-368, 2002

23. Van Linden AA, Baturin D, Ford JB, Fosmire SP, Gardner L, Korch C, Reigan P and Porter CC: Inhibition of weel sensitizes cancer cells to antimetabolite chemotherapeutics in vitro and in vivo, independent of p53 functionality. Mol Cancer Ther 12: 2675-2684, 2013.
24. Guertin AD, Li J, Liu Y, Hurd MS, Schuller AG, Long B, Hirsch HA, Feldman I, Benita Y, Toniatti C, et al: Preclinical evaluation of the WEE1 inhibitor MK-1775 as single-agent anticancer therapy. Mol Cancer Ther 12: 1442-1452, 2013.

25. Kreahling JM, Gemmer JY, Reed D, Letson D, Bui M and Altiok S: MK1775, a selective Weel inhibitor, shows single-agent antitumor activity against sarcoma cells. Mol Cancer Ther 11: 174-182, 2012.

26. Fischer von Weikersthal L, Schalhorn A, Stauch M, Quietzsch D, Maubach PA, Lambertz H, Oruzio D, Schlag R, Weigang-Köhler K, Vehling-Kaiser U, et al: Phase III trial of irinotecan plus infusional 5-fluorouracil/folinic acid versus irinotecan plus oxaliplatin as first-line treatment of advanced colorectal cancer. Eur J Cancer 47: 206-214, 2011.

27. Sun M, Zhang Q, Yang X, Qian SY and Guo B: Vitamin D enhances the efficacy of irinotecan through miR-627-mediated inhibition of intratumoral drug metabolism. Mol Cancer Ther 15: 2086-2095, 2016.

28. Quackenbush KS, Bagby S, Tai WM, Messersmith WA, Schreiber A, Greene J, Kim J, Wang G, Purkey A, Pitts TM, et al: The novel tankyrase inhibitor (AZ1366) enhances irinotecan activity in tumors that exhibit elevated tankyrase and irinotecan resistance. Oncotarget 7: 28273-28285, 2016. 